\title{
The Perception of Landscape Style: a Cross-cultural Comparison
}

\author{
BYOUNG-E YANG ${ }^{1}$ and RACHEL KAPLAN ${ }^{2}$ \\ ${ }^{1}$ Graduate School of Environmental Studies, Seoul National University, Kwanak-ku, Seoul (Korea) \\ ${ }^{2}$ School of Natural Resources, University of Michigan, Ann Arbor, MI 48109(U.S.A.)
}

(Accepted for publication 8 December 1989)

\begin{abstract}
Yang, B. and Kaplan, R., 1990. The perception of landscape style: a cross-cultural comparison. Landscape Urban Plann., 19: 251-262.

The perception of landscape style has received little attention. The present study explores this in a cross-cultural context both with respect to the landscapes (Korean, Japanese and Western) and in terms of the individual samples (Korean and Western). Preference ratings of 40
\end{abstract}

scenes taken in Korea provided the data for category-identifying methods to extract common perceptual groupings. The four distinct categories that emerged for both the Korean and the Western samples had strong similarities. They reflect a combination of landscape style and landscape content, with those based on more natural forms preferred to the more formal, linear categories.

\section{INTRODUCTION}

Throughout history, nations have developed unique landscape styles as a result of diverse factors including climate, geography, cultural heritage and the tastes of the residents. It is not unusual, however, to find examples of foreign landscape styles in many countries. With advances in transportation and communication, such transplants have become more prevalent. In Korea, for example, both Japanese and Western landscape styles are common in addition to the indigenous Korean style.
Landscape style is defined here as the typical mode of esthetic organization which characterizes the different kinds of landscape design originating in different countries or periods in the past (Hubbard and Kimball, 1917). Oriental landscape style, including Korean and Japanese landscape style, is characterized by asymmetry and non-geometrical form while Western landscape style has traditionally been based on the principles of geometry and symmetry. The differences between Korean and Japanese landscape styles can be found in terms of the lay-out of space, the use of plants, the 
use of rock and stone, the use of water and the manipulation of depth (see Yang, 1988; Tsu, 1988 , for the comparisons between landscape styles ).

Research on landscape preference has generally focused on landscape elements in the scene rather than landscape style. The focus of the current study is on how landscape style is perceived, including both the native style and exotic versions. Furthermore, these perceptions are considered both for the native population as well as for foreign tourists. The context for study is Korea and thus the comparisons include both Western and nonWestern influences. While, to the best of our knowledge, style has not been studied previously, cultural influences on landscape preference have received attention.

Some researchers have stressed culture as the pre-eminent determinant of preference (e.g. Lowenthal, 1968; Tuan, 1973). Much of the work that follows this line of argument explicitly or implicitly indicates that the influences of culture are so great as to preclude major similarities in preference across societies. Other researchers have provided support for strong cross-cultural similarity in esthetic judgments of various landscapes ranging from interior landscapes to natural landscapes (e.g. Shafer and Tooby, 1973; Berlyne et al., 1974; Ulrich, 1977; Kwok, 1979; Tips and Savasdisara, 1986). Despite an abundance of literature concerning landscape preferences, only a few studies have dealt with the comparison of Western and non-Western groups.

Previous cross-cultural comparisons have focused on preference mostly for natural landscapes, such as forests and coastal landscapes, and have found strong similarities using correlations between ratings (e.g. Zube and Mills, 1976; Zube and Pitt, 1981; Buhyoff et al., 1983). Kaplan and Herbert (1987, 1988), however, showed that some important crosscultural differences can be missed in such analyscs. Their studies pointed to differences in the perception of the landscape, although prefer- ence ratings were highly correlated. In other words, even if the relative preferences for different scenes are highly similar, the patterns among the ratings may be distinct (Kaplan, 1985).

The current study, focusing on designed landscapes, involves cross-cultural comparison in two senses, each including both Western and non-Western influences. The landscape styles that are studied include both the predominant Korean style, as well as Japanese and Western landscape styles found within the Korean environment. Furthermore, study participants included both Korean citizens and Western tourists.

The focus of the study is on the perception of landscape style. To what degree is the combination of factors that comprise a coherent style salient in the way individuals experience the landscape? Is the underlying categorization of the environment based on style or is it more strongly influenced by the objects in the scene? While participants were asked to rate scenes in terms of preference, these ratings were subsequently used as a vehicle for studying such categorization.

\section{METHODS}

\section{Participants}

The sample included three groups: Korean citizens, Korean students and Western tourists. All participants were over 18 yea $a_{1}$ of age. Selection of the citizen group was achieved through a multistage random sampling process to represent a wide variety of Seoul's citizens.

At the first stage, the $17 \mathrm{Gu}$, the la gest administrative district classification in Seoul, were divided into three groups based on the major housing type of each: (a) apartmentdominant $\mathrm{Gu}$; (b) detached house-dominant $\mathrm{Gu}$; (c) mixed housing-dominant $\mathrm{Gu}$ in which the proportion of apartment housing is roughly equal to that of detached housing. Six Gu were then selected randomly, with two Gu from each 
group. At the second stage, 18 Dong, the second largest administrative classification, were selected randomly with three Dong from each selected Gu. Random selection was made at the third stage to obtain six Tong (a subdivision of the Dong) from each selected Dong, which resulted in selecting 108 Tong in total. At the fourth stage, four Ban (the smallest administrative district) were chosen randomly from each selected Tong in the cases of detached house-dominant $\mathrm{Gu}$ and mixed housing-dominant $\mathrm{Gu}$. In the case of apartment-dominant $\mathrm{Gu}$, two floors were selected randomly from each selected Tong. At the fifth stage, the random selection of one household was made from each selected Ban in the cases of detached house-dominant $\mathrm{Gu}$ and mixed housing-dominant $\mathrm{Gu}$. In the case of apartment-dominant $\mathrm{Gu}$, two households were selected from each selected floor. At the final stage, a sample was selected from the families of selected households. The sample consisted of 415 individuals, representing a $96 \%$ response rate.

The second sample included 135 students at Seoul National University. Of these, 29 students were majoring in landscape architecture or architecture and 26 students were employed to conduct the survey of citizens and tourists; the others constituted a random sample of students at the Central Library of the University. Here too $96 \%$ of those who were asked agreed to participate in the study.

Sample selection of Western tourists involved approaching visitors, on a random basis, at the historic palaces, Changdok Palace (Secret Garden ), Kyongbok Palace and Doksu Palace, in Seoul. All 110 tourists who were asked agreed to participate.

\section{Procedure}

All the photographs were taken in Korea, mostly in Seoul. The 40 scenes comprising the photo-questionnaire represented three landscape styles: Korean, Japanese and Western and four landscape qualities: lay-out of space, use of landscape plants, use of stones and rocks, and use of water. For each combination of landscape style and quality, there were four scenes, with the exception of Japanese "layout of space" and Western "stones and rocks" for which there were none.

The process of scene selection involved several successive stages with cooperation from 15 Korean professors and experts in landscape architecture, as well as a panel of American professionals. While each scene was selected to reflect a specific landscape quality, other qualities are also present. Thus scenes representing the "lay-out of space" necessarily also include rock elements or water or vegetation.

The black and white photographs were printed in booklets with sur scenes on each page. Participants were asked to rate each scene in terms of their preference and degree of familiarity, using 5-point rating scales. Two sets of photo-questionnaires were printed, varying in the order of the scenes. The initial order was based on a random sequence in terms of landscape style and qualities. The second order was the reverse of the first.

\section{RESULTS}

Following the category-identifying methods (CIMs) used in many previous studies ( $\mathrm{Ka}$ plan and Kaplan, 1989), separate analyses were performed for the preference ratings of the Korean samples and the Western sample. Two approaches were used in these analyses: the Guttman-Lingoes Smallest Space Analysis III (SSA-III), a non-metric factor analysis (Lingoes, 1972) and ICLUST Hierarchical Cluster Analysis (Kulik et al., 1970). These analyses aid in the determination of the underlying perceptual categories. The following criteria were used in the identification of categories: (1) loadings that were at least $0.40 ;(2)$ scenes with loadings $>0.40$ on more than one category were omitted; (3) a category must be defined by at least three scenes; (4) eigenvalues $>1.0$.

For both the Korean-based and the Western- 


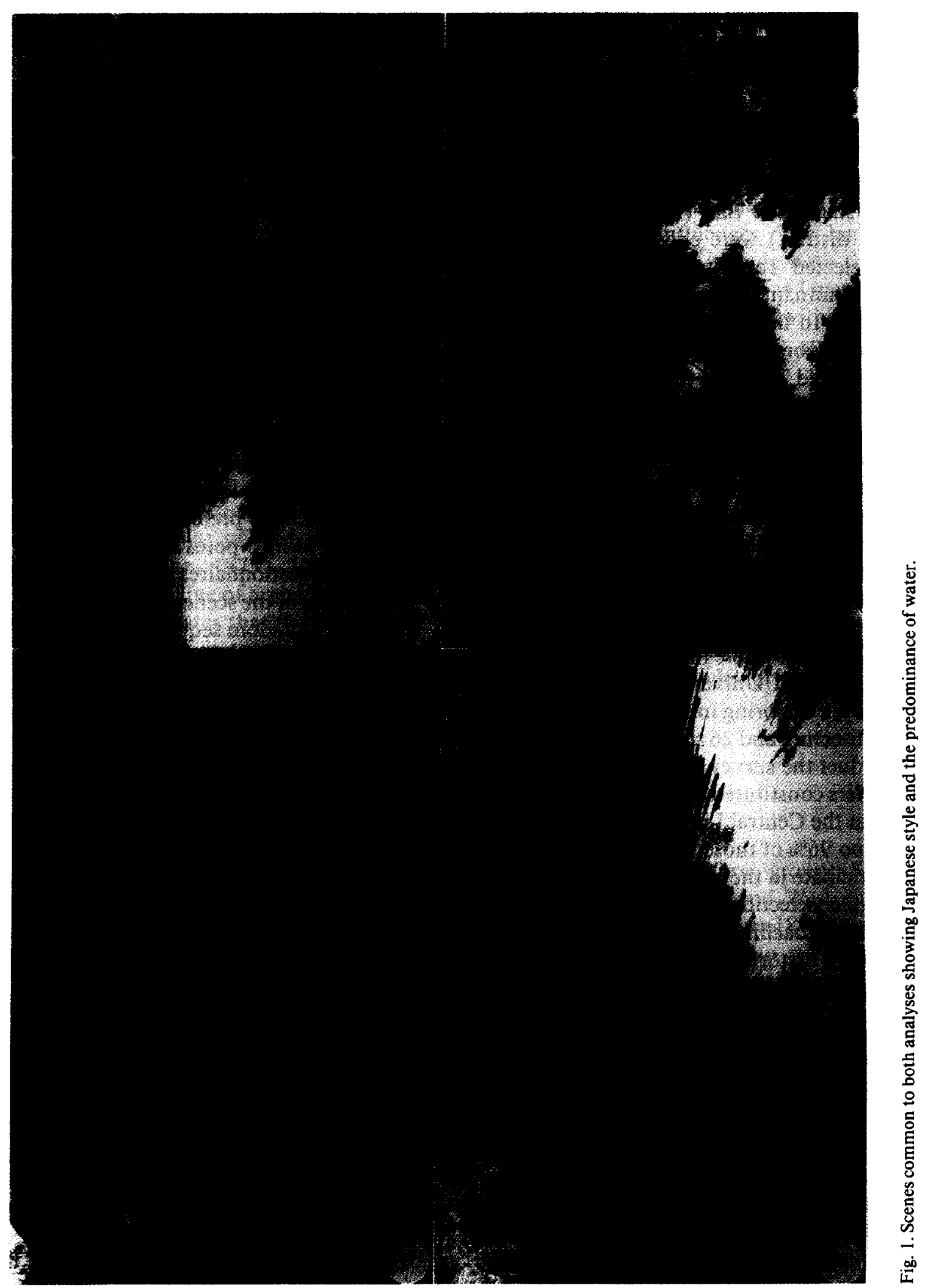




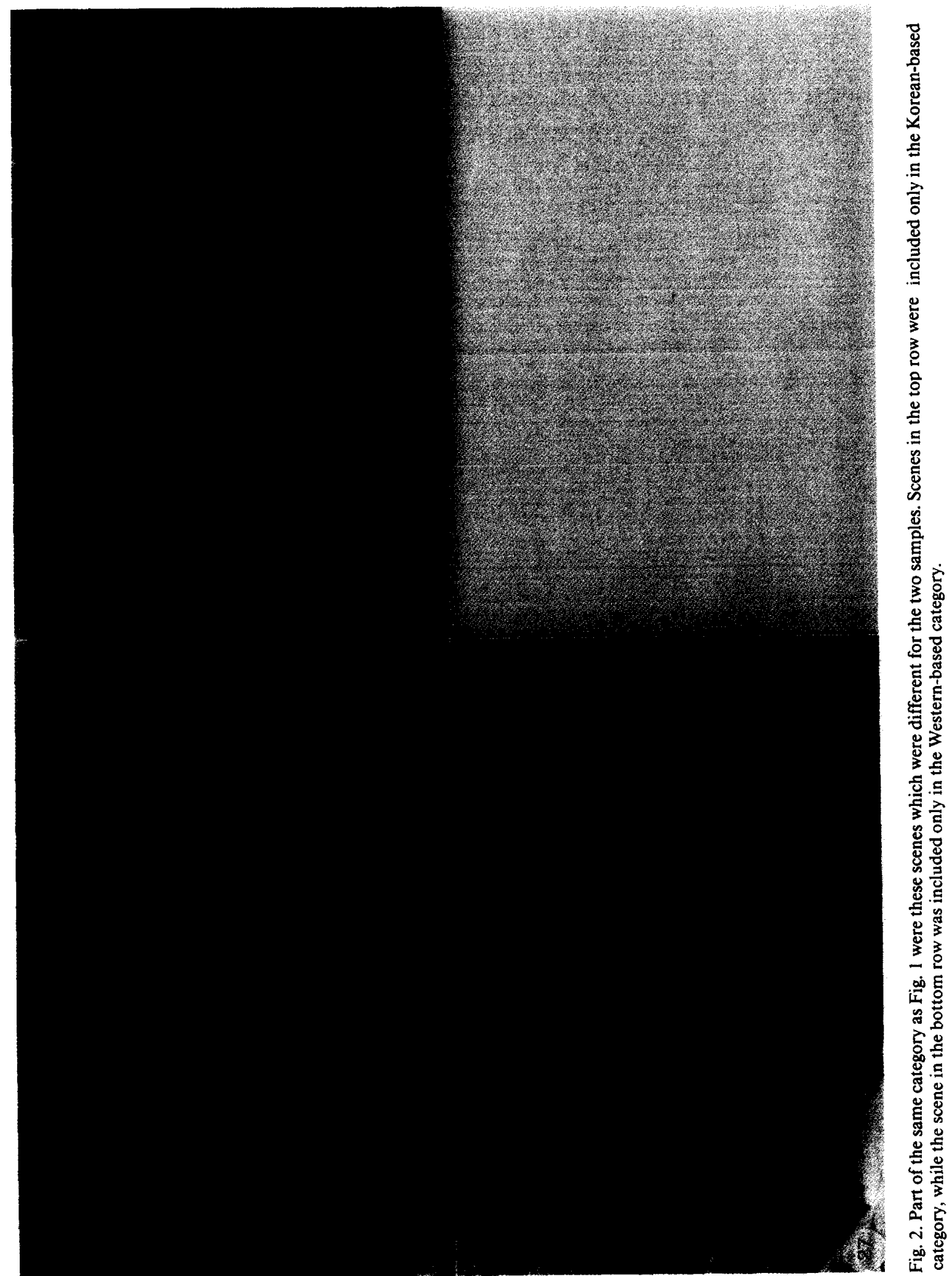




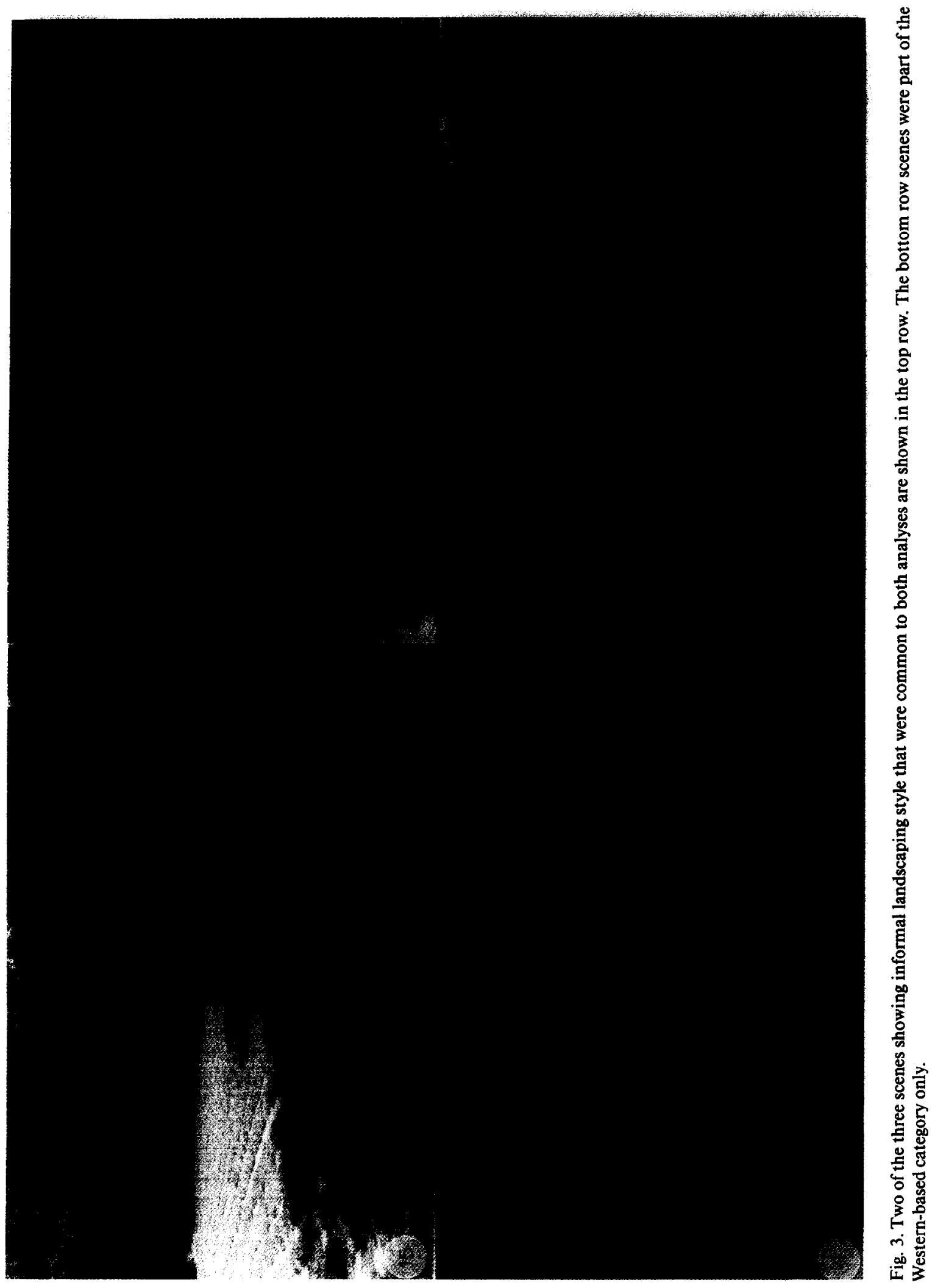




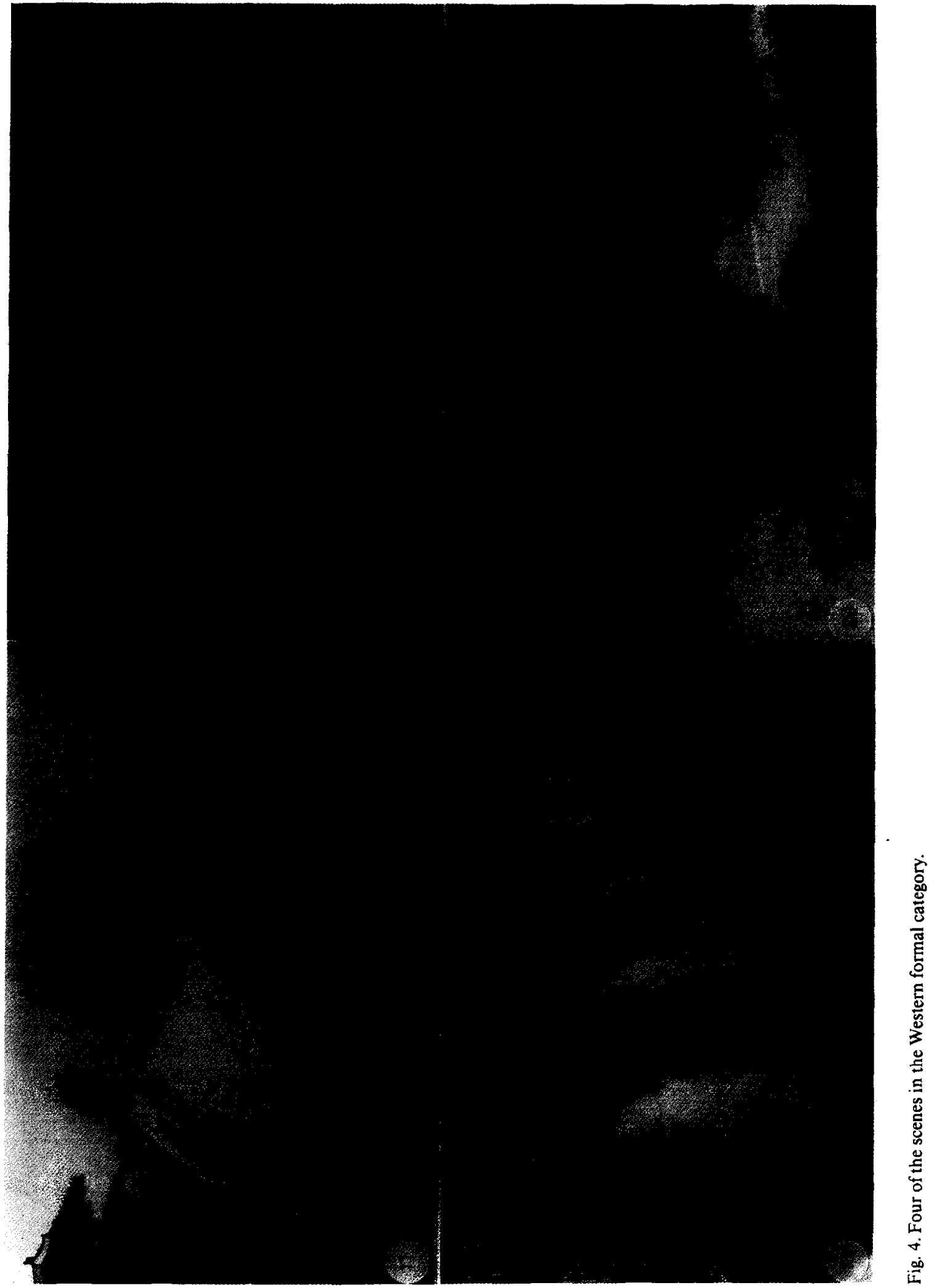




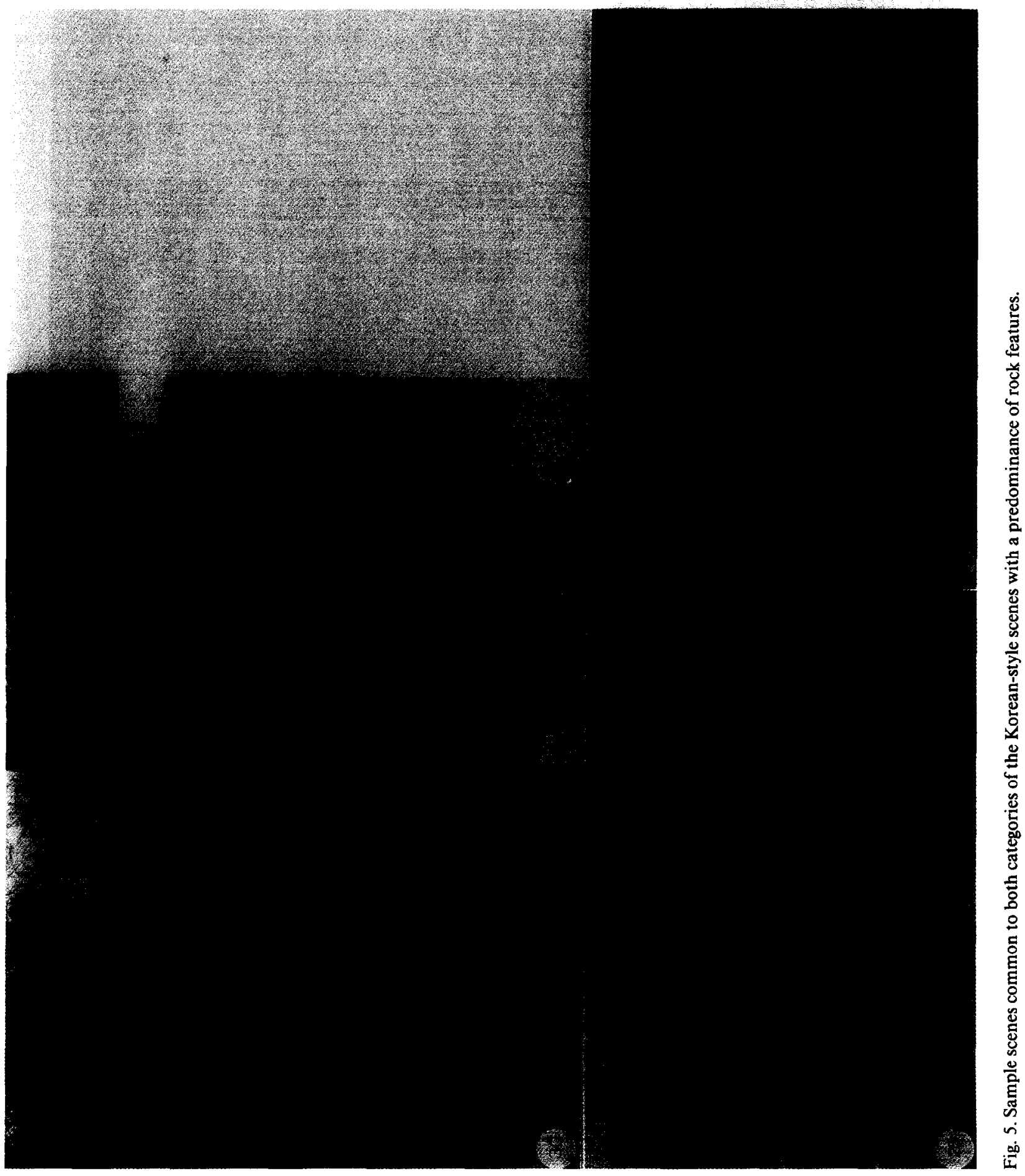




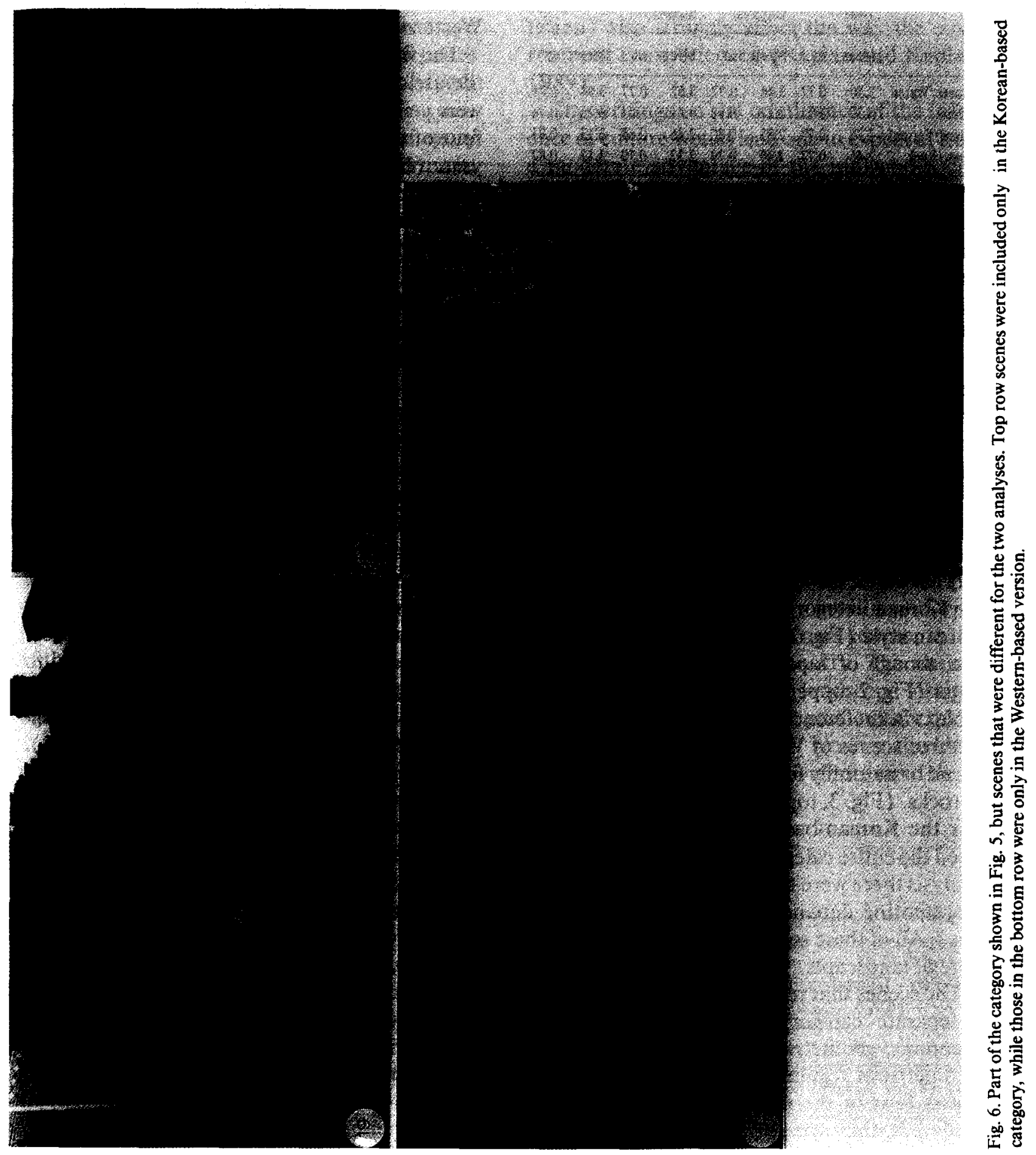


TABLE 1

Mean preference ratings and standard deviations for perceptual categories

\begin{tabular}{|c|c|c|c|c|c|c|c|c|}
\hline & \multicolumn{6}{|c|}{ Korean } & \multicolumn{2}{|c|}{ Western } \\
\hline & \multicolumn{2}{|l|}{ Total } & \multicolumn{2}{|c|}{ Citizens } & \multicolumn{2}{|c|}{ Students } & \multirow[b]{2}{*}{ Mean } & \multirow[b]{2}{*}{ sd } \\
\hline & Mean & sd & Mean & sd & Mean & sd & & \\
\hline Japanese/Water & 3.50 & 0.77 & 3.46 & 0.77 & 3.62 & 0.77 & 3.94 & 0.71 \\
\hline Informal & 3.30 & 0.95 & 3.27 & 0.93 & 3.41 & 1.01 & 3.25 & 0.76 \\
\hline Western/Formal & 2.75 & 0.78 & 2.85 & 0.80 & 2.46 & 0.64 & 3.13 & 0.93 \\
\hline Korean/Rock & 2.46 & 0.77 & 2.49 & 0.79 & 2.37 & 0.70 & 3.12 & 0.82 \\
\hline
\end{tabular}

based analyses, four categories emerged. Since these show strong similarities it is useful to discuss the two sets of results jointly.

The most preferred category in each case consisted, for the most part, of water scenes with distinctly Japanese influence. Of the six scenes common to both analyses all were of Japanese style (Fig. 1) with water as part of the scene. In many instances, scenes in this category show water surrounded by vegetation, with the trees reflecting in the water. The additional scenes in the Western category (Fig. 2, bottom row) reflected the Japanese style, but had no water. Of the two additional scenes in the Korean category, the one with water is of Korean style (Fig. 2, upper left) and the other one, though of Japanese style, does not have water (Fig. 2, upper right).

Next in preference was a category consisting of three scenes of Western style and featuring round or naturally formed trees with open lawn or rocks. (Fig. 3, top row, shows two of these.) For the Korean-based analysis, these constituted the entire category; for the Western-based analysis there were two additional scenes, both representing Japanese style (Fig. 3, bottom row ). All of these scenes represent an informal style of landscape.

The scenes that reflect a formal style formed a separate category. These included water fountains, geometric form of vegetation and symmetric or regular lay-out of space. Figure 4 shows four of the eight scenes that were included in this category in both the Korean- based and Western-based analyses. (One additional scene was included in the Korean analysis, but did not quite reach the 0.40 loading criterion for the Western sample.) All scenes in this category were designated as Western style.

The final category consisted of scenes where the use of rocks was a dominant feature; these were generally of Korean style. Figure 5 shows four of the nine scenes common to both analyses. (In both analyses one of the common scenes depicted a traditional Japanese lantern.) The Korean-based category included four additional scenes; Fig. 6, top row, shows two of these. Of the three scenes included only in the Western-based category, two included water (e.g. Fig. 6, lower left) and one was of Japanese style (Fig. 6, lower right).

Table 1 shows the mean preference ratings for the categories based on the Western and combined Korean samples. While the ordinal position of the four categories is comparable for the two groups, the magnitudes of the preferences are quite different. The ratings by the Western tourists are, in general, far more favorable than those of the Korean participants and do not differ from each other to as great an extent. For the Western sample, the most preferred category is significantly preferred to each of the three others. For the Korean sample as a whole, the preference means are all significantly different from each other $(P<0.01)$. Table 1 also shows the means for the two Korean groups, citizens and students. Here again, the ordinal positions remain constant. The two groups differ significantly in their preference of two of the categories: the students prefer the Japanese/Water scenes and the citizens are more favorable towards the Western Formal category.

With regard to the relationship between preference and familiarity, the correlation was substantial for both Korean $(r=0.81)$ and Western $(r=0.51)$ samples. 


\section{DISCUSSION AND CONCLUSIONS}

The cross-cultural similarity in these perceptual categorizations is striking. While the results for the two samples are not identical, the differences are subtle. For both the Korean and Western groups however the four categories that emerged reflect a combination of landscape style and landscape elements. For neither sample, for example, was there a category consisting solely of water scenes. Furthermore, the category in which water was a dominant attribute included none of the photographs with dynamic water. Similarly, none of the categories was a pure reflection of style. While the category with the water scenes also strongly reflected Japanese style, it included only a minority of the Japanese style scenes in the photoquestionnaire.

In terms of landscape style, the four categories suggest that both groups perceive a Japanese style that is distinct from a Korean style. A "Western" style however did not emerge as a single coherent style. Rather, the Western scenes were divided into two discrete categories representing the more formal traditions of French and Italian gardens, and the more informal arrangements which originated in 18 th century England and which were, in turn, influenced by reports sent home by missionaries and traders in China (Newton, 1971).

In terms of the landscape qualities included in the study, the four categories suggest that water and rock are important perceptual components, with distinct perceptual categories dominated by each of these. Furthermore, whether vegetation is sparse or ample also played an important role; the categories differ in this respect as well.

The arrangement of the space and the choice of forms used in the lay-out seem to have the greatest impact on perceptual distinctions. Two of the categories, Korean/Rock and Western Formal, are dominated by more rectangular arrangements while the other two, Japanese/ Water and Informal, entail rounder, softer shapes. The fact that there are two separate categories in each case however implies that more than the forms are critical in the perceptual process. The differences in the contents of the scenes (affected by water, rock and vegetation) also strongly affect the way the environment is experienced (Kaplan and Kaplan, 1989).

The writings on the dominance of the influence of culture would lead one to expect at least a moderate advantage of the indigenous cultural style in preference. The particularly low preference ratings for their own cultural style on the part of the Korean sample thus comes as a surprise. In the present study, both cultural groups were consistent in their greater preference for the categories that involve more natural forms rather than more rectangular or formal designs. We are not aware of previous research on environmental preference that addressed this question.

While the findings are useful in the context of cross-cultural comparison and in terms of understanding the underlying bases of perception, they also have implications for landscape design, especially in the Korean context. The preference for natural and curvilinear forms and for asymmetric lay-outs runs counter to the cultural tradition of the country. The importation of formal Western landscape design, however, would seem to be particularly questionable given these results. Neither the local population nor the Western visitors rated these scenes with great favor.

\section{REFERENCES}

Berlyne, D.E., Robbins, M.C. and Thompson, R., 1974. A cross-cultural study of exploratory and verbal responses to visual patterns varying in complexity. In: D.E. Berlyne (Editor), Studies in the New Experimental Aesthetics: Steps toward an Objective Psychology of Aesthetic Appreciation. Wiley, New York, pp. 259-278.

Buhyoff, G.J., Wellman, J.D., Koch, N.E., Gauthier, L. and Hultman, S., 1983. Landscape preference metrics: an international comparison. J. Environ. Manage., 16: 181-190.

Hubbard, H.V. and Kimball, T., 1917. An Introduction to the Study of Landscape Design. Macmillan, New York. 
Kaplan, R., 1985. The analysis of perception via preference: a strategy for studying how the environment is experienced. Landscape Plann., 12: 161-176.

Kaplan, R. and Herbert, E.J., 1987. Cultural and sub-cultural comparisons in preferences for natural settings. Landscape Urban Plann., 14: 281-293.

Kaplan, R. and Herbert, E.J., 1988. Familiarity and preference: a cross-cultural analysis. In: J.L. Nasar (Editor), Environmental Aesthetics: Theory, Research and Applications. Cambridge, New York, pp. 379-389.

Kaplan, R. and Kaplan, S., 1989. The Experience of Nature: A Psychological Perspective. Cambridge, New York.

Kulik, J.A., Revelle, W.R. and Kulik, C.L.C., 1970. Scale construction by hierarchical cluster analysis. University of Michigan, (unpublished).

Kwok, K., 1979. Semantic evaluation of perceived environment: a cross-cultural replication. Man-Environ. Syst., 9: 243-249.

Lingoes, J.C., 1972. A general survey of the Guttman-Lingoes nonmetric program series. In: R.N. Shepard, A.K. Romney and S.B. Nerlove (Editors), Multidimensional Scaling. Vol. 1. Seminar Press, New York, pp. 52-68.

Lowenthal, D., 1968. The American Scene. Geogr. Rev., 58: 61-88.

Newton, N.T., 1971. Design on the Land. Belknap Press, Cambridge, MA, U.S.A.
Shafer, E.L. and Tooby, M., 1973. Landscape preferences: an international replication. J. Leisure Res., 5: 60-65.

Tips, W.E.J. and Savasdisara, T., 1986. The influence of the environmental background of subjects on their landscape preference evaluation. Landscape Plann., 13: 125-133.

Tsu, F.Y., 1988. Landscape Design in Chinese Gardens. McGraw-Hill, New York.

Tuan, Y.-F., 1973. Visual blight: exercises in interpretation. In: Visual Blight in America. Commission of College Geography Resource Paper No. 23, Association of American Geographers, pp. 23-27.

Ulrich, R.S., 1977. Visual landscape preference: a model and application. Man-Environ. Syst., 7: 279-293.

Yang, B., 1988. A cross-cultural comparison of preference for Korean, Japanese and Western landscape styles. $\mathrm{PhD}$ dissertation, University of Michigan, (unpublished).

Zube, E.H. and Mills, L.V., Jr., 1976. Cross-cultural exploration in landscape perception. In: E.H. Zube (Editor), Studies in Landscape Perception. Institute for Man and Environment, University of Massachusetts, Amherst, MA, pp. 167-174.

Zube, E.H. and Pitt, D.G., 1981. Cross-cultural perceptions of scenic and heritage landscapes. Landscape Plann., 8: 69-87. 